\title{
Nurse Responses to Physiologic Monitor Alarms on a General Pediatric Unit
}

\author{
Amanda C Schondelmeyer, MD, MSc, ${ }^{1,2,3, *}$ Nancy M Daraiseh, PhD, ${ }^{2,3,4}$ Brittany Allison, BSN, RN, CPN, ${ }^{4}$ Cindi Acree, DNP, APRN, ${ }^{5}$ \\ Allison M Loechtenfeldt, BS, ${ }^{1}$ Kristen M Timmons, BS, BA, ${ }^{1}$ Colleen Mangeot, MS, ${ }^{6}$ Patrick W Brady, MD, MSc ${ }^{1,2,3}$
}

'Division of Hospital Medicine, Cincinnati Children's Hospital Medical Center, Cincinnati, Ohio; ${ }^{2} J a m e s$ M. Anderson Center for Health Systems Excellence, Cincinnati Children's Hospital Medical Center, Cincinnati, Ohio; ${ }^{3}$ Department of Pediatrics, University of Cincinnati College of Medicine, Cincinnati, Ohio; ${ }^{4}$ Department of Patient Services, Cincinnati Children's Hospital Medical Center, Cincinnati, Ohio; ${ }^{5}$ Division of Neonatology, Cincinnati Children's Hospital Medical Center, Cincinnati, Ohio; ${ }^{6}$ Division of Biostatistics and Epidemiology, Cincinnati Children's Hospital Medical Center, Cincinnati, Ohio.

BACKGROUND: Hospitalized children generate up to 152 alarms per patient per day outside of the intensive care unit. In that setting, as few as $1 \%$ of alarms are clinically important. How nurses make decisions about responding to alarms, given an alarm's low specificity for detecting clinical deterioration, remains unclear.

OBJECTIVE: Our objective was to describe how bedside nurses think about and act upon monitor alarms for hospitalized children.

DESIGN, SETTING, PARTICIPANTS: This was a qualitative study that involved the direct observation of nurses working on a general pediatric unit at a large children's hospital.

MEASUREMENTS: We used a structured tool that included predetermined categories to assess nurse responses to monitor alarms. Data on alarm frequency and type were pulled from bedside monitors.
RESULTS: We conducted 61.3 patient-hours of observation with nine nurses, in which we documented 207 nurse responses to patient alarms. For $67 \%$ of alarms heard outside of the room, the nurse decided not to respond without further assessment. Nurses most commonly cited reassuring clinical context (eg, medical team in room), as the rationale for alarm nonresponse. The nurse deemed clinical intervention necessary in only 14 (7\%) of the observed responses.

CONCLUSION: Nurses rely on clinical and contextual details to determine how to respond to alarms. Few of the alarm responses in our study resulted in a clinical intervention. These findings suggest that multiple systemlevel and educational interventions may be necessary to improve the efficacy and safety of continuous monitoring. Journal of Hospital Medicine 2019;14:602-606. (c) 2019 Society of Hospital Medicine



larms from bedside continuous physiologic monitors (CPMs) occur frequently in children's hospitals and can lead to harm. Recent studies conducted in children's hospitals have identified alarm rates of up to 152 alarms per patient per day outside of the intensive care unit, ${ }^{1-3}$ with as few as $1 \%$ of alarms being considered clinically important. ${ }^{4}$ Excessive alarms have been linked to alarm fatigue, when providers become desensitized to and may miss alarms indicating impending patient deterioration. Alarm fatigue has been identified by national patient safety organizations as a patient safety concern given the risk of patient harm. ${ }^{5-7}$ Despite these concerns, CPMs are routinely used: up to $48 \%$ of pediatric patients in nonintensive care units at children's hospitals are monitored. ${ }^{2}$

Although the low number of alarms that receive responses has been well-described, ${ }^{8,9}$ the reasons why clinicians do or do not respond to alarms are unclear. A study conducted in

*Corresponding Author: Amanda Schondelmeyer, MD, MSc; E-mail: amanda. schondelmeyer@cchmc.org; Telephone: 513-803-9158

Published online first June 11, 2019.

Received: December 22, 2018; Revised: April 11, 2019; Accepted: April 15, 2019

(c) 2019 Society of Hospital Medicine DOI 10.12788/jhm.3234 an adult perioperative unit noted prolonged nurse response times for patients with high alarm rates..$^{10} \mathrm{~A}$ second study conducted in the pediatric inpatient setting demonstrated a dose-response effect and noted progressively prolonged nurse response times with increased rates of nonactionable alarms. ${ }^{4,11}$ Findings from another study suggested that underlying factors are highly complex and may be a result of excessive alarms, clinician characteristics, and working conditions (eg, workload and unit noise level). ${ }^{12}$ Evidence also suggests that humans have difficulty distinguishing the importance of alarms in situations where multiple alarm tones are used, a common scenario in hospitals. ${ }^{13,14}$ Understanding the factors that contribute to clinicians responding or not responding to CPM alarms will be crucial for addressing this serious patient safety issue.

An enhanced understanding of why nurses respond to alarms in daily practice will inform intervention development and improvement work. In the long term, this information could help improve systems for monitoring pediatric inpatients that are less prone to issues with alarm fatigue. The objective of this qualitative study, which employed structured observation, was to describe how bedside nurses think about and act upon bedside monitor alarms in a general pediatric inpatient unit. 


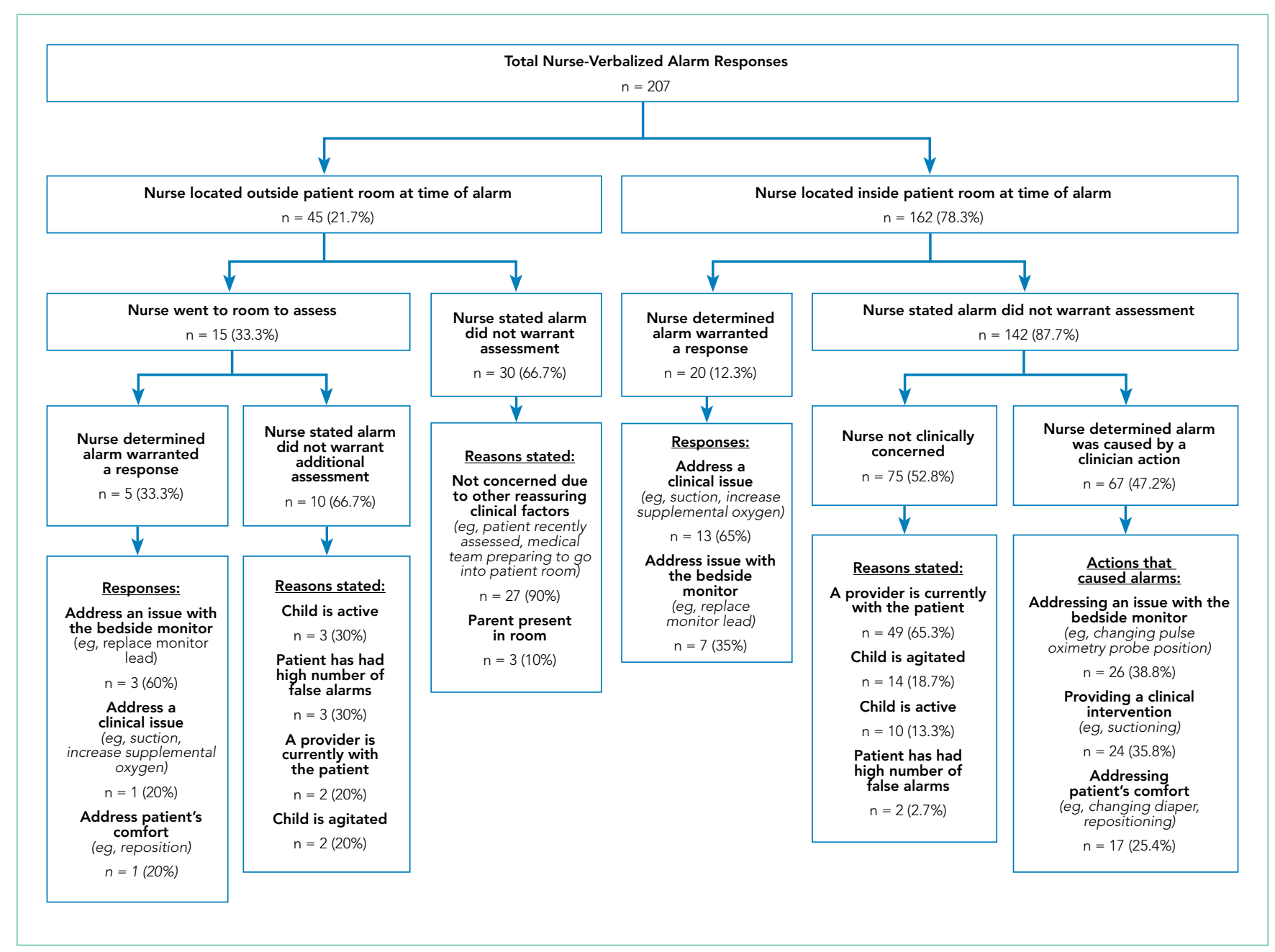

FIG. Nurse-Verbalized Responses to Alarms.

\section{METHODS}

\section{Study Design and Setting}

This prospective observational study took place on a 48-bed hospital medicine unit at a large, freestanding children's hospital with $>650$ beds and >19,000 annual admissions. General Electric (Little Chalfont, United Kingdom) physiologic monitors (models Dash 3000, 4000, and 5000) were used at the time of the study, and nurses could be notified of monitor alarms in four ways: First, an in-room auditory alarm sounds. Second, a light positioned above the door outside of each patient room blinks for alarms that are at a "warning" or "critical level" (eg ventricular tachycardia or low oxygen saturation). Third, audible alarms occur at the unit's central monitoring station. Lastly, another staff member can notify the patient's nurse via in-person conversion or secure smart phone communication. On the study unit, CPMs are initiated and discontinued through a physician order.

This study was reviewed and approved by the hospital's institutional review board.

\section{Study Population}

We used a purposive recruitment strategy to enroll bedside nurses working on general hospital medicine units, stratified to ensure varying levels of experience and primary shifts (eg, day vs night). We planned to conduct approximately two observations with each participating nurse and to continue collecting data until we could no longer identify new insights in terms of responses to alarms (ie, thematic saturation ${ }^{15}$ ). Observations were targeted to cover times of day that coincided with increased rates of distraction. These times included just prior to and after the morning and evening change of shifts (7:00 AM and 7:00 PM), during morning rounds (8:00 AM-12:00 PM), and heavy admission times (12:00 PM-10:00 PM). After written informed consent, a nurse was eligible for observation during his/her shift if he/she was caring for at least one monitored patient. Enrolled nurses were made aware of the general study topic but were blinded to the study team's hypotheses.

\section{Data Sources}

Prior to data collection, the research team, which consisted of physicians, bedside nurses, research coordinators, and a human factors expert, created a system for categorizing alarm responses. Categories for observed responses were based on the location and corresponding action taken. Initial categories 
were developed a priori from existing literature and expanded through input from the multidisciplinary study team, then vetted with bedside staff, and finally pilot tested through $>4$ hours of observations, thus producing the final categories. These categories were entered into a work-sampling program (WorkStudy by Quetech Ltd., Waterloo, Ontario, Canada) to facilitate quick data recording during observations.

The hospital uses a central alarm collection software (BedMasterEx by Anandic Medical Systems, Feuerthalen, Switzerland), which permitted the collection of date, time, trigger (eg, high heart rate), and level (eg, crisis, warning) of the generated CPM alarms. Alarms collected are based on thresholds preset at the bedside monitor. The central collection software does not differentiate between accurate (eg, correctly representing the physiologic state of the patient) and inaccurate alarms.

\section{Observation Procedure}

At the time of observation, nurse demographic information (eg, primary shift worked and years working as a nurse) was obtained. A brief preobservation questionnaire was administered to collect patient information (eg, age and diagnosis) and the nurses' perspectives on the necessity of monitors for each monitored patient in his/her care.

The observer shadowed the nurse for a two-hour block of his/her shift. During this time, nurses were instructed to "think aloud" as they responded to alarms (eg, "I notice the oxygen saturation monitor alarming off, but the probe has fallen off"). A trained observer (AML or KMT) recorded responses verbalized by the nurse and his/her reaction by selecting the appropriate category using the work-sampling software. Data were also collected on the vital sign associated with the alarm (eg, heart rate). Moreover, the observer kept written notes to provide context for electronically recorded data. Alarms that were not verbalized by the nurse were not counted. Similarly, alarms that were noted outside of the room by the nurse were not classified by vital sign unless the nurse confirmed with the bedside monitor. Observers did not adjudicate the accuracy of the alarms. The session was stopped if monitors were discontinued during the observation period. Alarm data generated by the bedside monitor were pulled for each patient room after observations were completed.

\section{Analysis}

Descriptive statistics were used to assess the percentage of each nurse response category and each alarm type (eg, heart rate and respiratory rate). The observed alarm rate was calculated by taking the total number of observed alarms (ie, alarms noted by the nurse) divided by the total number of patient-hours observed. The monitor-generated alarm rate was calculated by taking the total number of alarms from the bedside-alarm generated data divided by the number of patient-hours observed.

Electronically recorded observations using the work-sampling program were cross-referenced with hand-written field notes to assess for any discrepancies or identify relevant events not captured by the program. Three study team mem-
TABLE. Patient Characteristics

\begin{tabular}{lc}
\hline Characteristics & ( $\mathbf{n}=35)$ \\
\hline $\begin{array}{l}\text { Age, } \mathrm{n}(\%) \\
\text { Under } 2 \text { years }\end{array}$ & $13(38.2)$ \\
2 years to 6 years & $10(29.4)$ \\
Greater than 7 years & $11(32.4)$ \\
\hline Female, $\mathrm{n}(\%)$ & $17(48.6)$ \\
\hline Diagnosis category, $\mathrm{n}(\%)$ & \\
Asthma & $2(5.7)$ \\
Bronchiolitis & $4(11.4)$ \\
BRUE & $3(8.6)$ \\
High risk therapy & $2(5.7)$ \\
Ingestion & $1(2.9)$ \\
Other & $4(11.4)$ \\
Other infectious & $2(5.7)$ \\
Other respiratory & $9(25.7)$ \\
Pneumonia & $6(17.1)$ \\
Postoperative & $1(2.9)$ \\
Sepsis & $1(2.9)$ \\
\hline Family present in room, $\mathrm{n}(\%)$ & $25(71.4)$ \\
\hline RN perspective on need for monitors, $\mathrm{n}(\%)$ & $29(82.9)$ \\
\hline
\end{tabular}

${ }^{a} n=34$

Abbreviations: BRUE, brief resolved unexplained event; RN, registered nurse.

bers (AML, KMT, and ACS) reviewed each observation independently and compared field notes to ensure accurate categorization. Discrepancies were referred to the larger study group in cases of uncertainty.

\section{RESULTS}

Nine nurses had monitored patients during the available observations and participated in 19 observation sessions, which included 35 monitored patients for a total of 61.3 patient-hours of observation. Nurses were observed for a median of two times each (range 1-4). The median number of monitored patients during a single observation session was two (range 1-3). Observed nurses were female with a median of eight years of experience (range 0.5-26 years). Patients represented a broad range of age categories and were hospitalized with a variety of diagnoses (Table). Nurses, when queried at the start of the observation, felt that monitors were necessary for 29 (82.9\%) of the observed patients given either patient condition or unit policy.

A total of 207 observed nurse responses to alarms occurred during the study period for a rate of 3.4 responses per patient per hour. Of the total number of responses, $45(21.7 \%)$ were noted outside of a patient room, and in 15 (33.3\%) the nurse chose to go to the room. The other 162 were recorded when the nurse was present in the room when the alarm activated. Of the 177 in-person nurse responses, 50 were related to a pulse oximetry alarm, 66 were related to a heart rate alarm, and 61 were related to a respiratory rate alarm. The most common 
observed in-person response to an alarm involved the nurse judging that no intervention was necessary ( $n=152,73.1 \%$ ). Only 14 (7\% of total responses) observed in-person responses involved a clinical intervention, such as suctioning or titrating supplemental oxygen. Findings are summarized in the Figure and describe nurse-verbalized reasons to further assess (or not) and then whether the nurse chose to take action (or not) after an alarm.

Alarm data were available for 17 of the 19 observation periods during the study. Technical issues with the central alarm collection software precluded alarm data collection for two of the observation sessions. A total of 483 alarms were recorded on bedside monitors during those 17 observation periods or 8.8 alarms per patient per hour, which was equivalent to 211.2 alarms per patient-day. A total of 175 observed responses were collected during these 17 observation periods. This number of responses was $36 \%$ of the number we would have expected on the basis of the alarm count from the central alarm software.

There were no patients transferred to the intensive care unit during the observation period. Nurses who chose not to respond to alarms outside the room most often cited the brevity of the alarm or other reassuring contextual details, such as that a family member was in the room to notify them if anything was truly wrong, that another member of the medical team was with the patient, or that they had recently assessed the patient and thought likely the alarm did not require any action. During three observations, the observed nurse cited the presence of family in the patient's room in their decision not to conduct further assessment in response to the alarm, noting that the parent would be able to notify the nurse if something required attention. On two occasions in which a nurse had multiple monitored patients, the observed nurse noted that if the other monitored patients were alarming and she happened to be in another patient's room, she would not be able to hear them. Four nurses cited policy as the reason a patient was on monitors (eg, patient was on respiratory support at night for obstructive sleep apnea).

\section{DISCUSSION}

We characterized responses to physiologic monitor alarms by a group of nurses with a range of experience levels. We found that most nurse responses to alarms in continuously monitored general pediatric patients involved no intervention, and further assessment was often not conducted for alarms that occurred outside of the room if the nurse noted otherwise reassuring clinical context. Observed responses occurred for $36 \%$ of alarms during the study period when compared with bedside monitor-alarm generated data. Overall, only 14 clinical interventions were noted among the observed responses. Nurses noted that they felt the monitors were necessary for $82.9 \%$ of monitored patients because of the clinical context or because of unit policy.

Our study findings highlight some potential contradictions in the current widespread use of CPMs in general pediatric units and how clinicians respond to them in practice. ${ }^{2}$ First, while nurses reported that monitors were necessary for most of their patients, participating nurses deemed few alarms clinically actionable and often chose not to further assess when they noted alarms outside of the room. This is in line with findings from prior studies suggesting that clinicians overvalue the contribution of monitoring systems to patient safety. ${ }^{16,17}$ Second, while this finding occurred in a minority of the observations, the presence of family members at the patient's bedside was cited by nurses as a rationale for whether they responded to alarms. While family members are capable of identifying safety issues, ${ }^{18}$ formal systems to engage them in patient safety and physiologic monitoring are lacking. Finally, clinical interventions or responses to the alerts of deteriorating patients, which best represented the original intent of CPMs, were rare and accounted for just $7 \%$ of the responses. Further work elucidating why physicians and nurses choose to use CPMs may be helpful to identify interventions to reduce inappropriate monitor use and highlight gaps in frontline staff knowledge about the benefits and risks of CPM use.

Our findings provide a novel understanding of previously observed phenomena, such as long response times or nonresponses in settings with high alarm rates. ${ }^{4,10}$ Similar to that in a prior study conducted in the pediatric setting, ${ }^{11}$ alarms with an observed response constituted a minority of the total alarms that occurred in our study. This finding has previously been attributed to mental fatigue, caregiver apathy, and desensitization. ${ }^{8}$ However, even though a minority of observed responses in our study included an intervention, the nurse had a rationale for why the alarm did or did not need a response. This behavior and the verbalized rationale indicate that in his/her opinion, not responding to the alarm was clinically appropriate. Study participants also reflected on the difficulties of responding to alarms given the monitor system setup, in which they may not always be capable of hearing alarms for their patients. Without data from nurses regarding the alarms that had no observed response, we can only speculate; however, based on our findings, each of these factors could contribute to nonresponse. Finally, while high numbers of false alarms have been posited as an underlying cause of alarm fatigue, we noted that a majority of nonresponse was reported to be related to other clinical factors. This relationship suggests that from the nurse's perspective, a more applicable framework for understanding alarms would be based on clinical actionability ${ }^{4}$ over physiologic accuracy.

In total, our findings suggest that a multifaceted approach will be necessary to improve alarm response rates. These interventions should include adjusting parameters such that alarms are highly likely to indicate a need for intervention coupled with educational interventions addressing clinician knowledge of the alarm system and bias about the actionability of alarms may improve response rates. Changes in the monitoring system setup such that nurses can easily be notified when alarms occur may also be indicated, in addition to formally engaging patients and families around response to alarms. Although secondary notification systems (eg, alarms transmitted to individual clinician's devices) are one solution, 
the utilization of these systems needs to be balanced with the risks of contributing to existing alarm fatigue and the need to appropriately tailor monitoring thresholds and strategies to patients.

Our study has several limitations. First, nurses may have responded in a way they perceive to be socially desirable, and studies using in-person observers are also prone to a Hawthorne-like effect, ${ }^{19-21}$ where the nurse may have tried to respond more frequently to alarms than usual during observations. However, given that the majority of bedside alarms did not receive a response and a substantial number of responses involved no action, these effects were likely weak. Second, we were unable to assess which alarms were accurately reflecting the patient's physiologic status and which were not; we were also unable to link observed alarm response to monitor-recorded alarms. Third, despite the use of silent observers and an actual, rather than a simulated, clinical setting, by virtue of the data collection method we likely captured a more deliberate thought process (so-called System 2 thinking) ${ }^{22}$ rather than the subconscious processes that may predominate when nurses respond to alarms in the course of clinical care (System 1 thinking). ${ }^{22}$ Despite this limitation, our study findings, which reflect a nurse's in-the-moment thinking, remain relevant to guiding the improvement of monitoring systems, and the development of nurse-facing interventions and education. Finally, we studied a small, purposive sample of nurses at a single hospital. Our study sample impacts the generalizability of our results and precluded a detailed analysis of the effect of nurse- and patient-level variables.

\section{CONCLUSION}

We found that nurses often deemed that no response was necessary for CPM alarms. Nurses cited contextual factors, including the duration of alarms and the presence of other providers or parents in their decision-making. Few (7\%) of the alarm responses in our study included a clinical intervention. The number of observed alarm responses constituted roughly a third of the alarms recorded by bedside CPMs during the study. This result supports concerns about the nurse's capacity to hear and process all CPM alarms given system limitations and a heavy clinical workload. Subsequent steps should include staff education, reducing overall alarm rates with appropriate monitor use and actionable alarm thresholds, and ensuring that patient alarms are easily recognizable for frontline staff.

Disclosures: The authors have no conflicts of interest to disclose.

Funding: This work was supported by the Place Outcomes Research Award from the Cincinnati Children's Research Foundation. Dr. Brady is supported by the Agency for Healthcare Research and Quality under Award Number
K08HS23827. The content is solely the responsibility of the authors and does not necessarily represent the official views of the Agency for Healthcare Research and Quality.

\section{References}

1. Schondelmeyer AC, Bonafide CP, Goel W, et al. The frequency of physiologic monitor alarms in a children's hospital. J Hosp Med. 2016;11(11):796-798. https://doi.org/10.1002/jhm.2612.

2. Schondelmeyer AC, Brady PW, Goel W, et al. Physiologic monitor alarm rates at 5 children's hospitals. J Hosp Med. 2018;13(6):396-398. https://doi. org/10.12788/jhm.2918.

3. Schondelmeyer AC, Brady PW, Sucharew $H$, et al. The impact of reduced pulse oximetry use on alarm frequency. Hosp Pediatr. In press.

4. Bonafide $\mathrm{CP}$, Lin R, Zander M, et al. Association between exposure to nonactionable physiologic monitor alarms and response time in a children's hospital. J Hosp Med. 2015;10(6):345-351. https://doi.org/10.1002/jhm.2331.

5. Siebig $S$, Kuhls $S$, Imhoff $M$, et al. Intensive care unit alarms--how many do we need? Crit Care Med. 2010;38(2):451-456. https://doi.org/10.1097/CCM. Ob013e3181cb0888.

6. Sendelbach S, Funk M. Alarm fatigue: a patient safety concern. AACNAdv Crit Care. 2013;24(4):378-386. https://doi.org/10.1097/NCl.0b013e3182a903f9.

7. Sendelbach S. Alarm fatigue. Nurs Clin North Am. 2012;47(3):375-382. https://doi.org/10.1016/j.cnur.2012.05.009.

8. Cvach M. Monitor alarm fatigue: an integrative review. Biomed Instrum Technol. 2012;46(4):268-277. https://doi.org/10.2345/0899-8205-46.4.268.

9. Paine CW, Goel W, Ely E, et al. Systematic review of physiologic monitor alarm characteristics and pragmatic interventions to reduce alarm frequency. J Hosp Med. 2016;11(2):136-144. https://doi.org/10.1002/jhm.2520.

10. Voepel-Lewis T, Parker ML, Burke $C N$, et al. Pulse oximetry desaturation alarms on a general postoperative adult unit: a prospective observational study of nurse response time. Int J Nurs Stud. 2013;50(10):1351-1358. https:// doi.org/10.1016/j.ijnurstu.2013.02.006.

11. Bonafide $\mathrm{CP}$, Localio $\mathrm{AR}$, Holmes $\mathrm{JH}$, et al. Video analysis of factors associated With response time to physiologic monitor alarms in a children's hospital. JAMA Pediatr. 2017;171(6):524-531. https://doi.org/10.1001/jamapediatrics.2016.5123.

12. Deb S, Claudio D. Alarm fatigue and its influence on staff performance. IIE Trans Healthc Syst Eng. 2015;5(3):183-196. https://doi.org/10.1080/19488300 .2015.1062065.

13. Mondor TA, Hurlburt J, Thorne L. Categorizing sounds by pitch: effects of stimulus similarity and response repetition. Percept Psychophys. 2003:65(1):107-114. https://doi.org/10.3758/BF03194787.

14. Mondor TA, Finley GA. The perceived urgency of auditory warning alarms used in the hospital operating room is inappropriate. Can $J$ Anaesth. 2003;50(3):221-228. https://doi.org/10.1007/BF03017788.

15. Fusch PI, Ness LR. Are we there yet? Data saturation in qualitative research. Qual Rep; 20(9), 2015:1408-1416.

16. Najafi N, Auerbach $A$. Use and outcomes of telemetry monitoring on a medicine service. Arch Intern Med. 2012;172(17):1349-1350. https://doi. org/10.1001/archinternmed.2012.3163.

17. Estrada CA, Rosman HS, Prasad NK, et al. Role of telemetry monitoring in the non-intensive care unit. Am J Cardiol. 1995;76(12):960-965. https://doi. org/10.1016/S0002-9149(99)80270-7.

18. Khan A, Furtak SL, Melvin P et al. Parent-reported errors and adverse events in hospitalized children. JAMA Pediatr. 2016;170(4):e154608. https://doi. org/10.1001/jamapediatrics.2015.4608.

19. Adair JG. The Hawthorne effect: a reconsideration of the methodological artifact. J Appl Psychol. 1984;69(2):334-345. https://doi.org/10.1037/00219010.69.2.334

20. Kovacs-Litman A, Wong K, Shojania KG, et al. Do physicians clean their hands? Insights from a covert observational study. J Hosp Med. 2016;11(12):862-864. https://doi.org/10.1002/jhm.2632.

21. Wolfe F, Michaud K. The Hawthorne effect, sponsored trials, and the overestimation of treatment effectiveness. J Rheumatol. 2010;37(11):2216-2220. https://doi.org/10.3899/jrheum.100497.

22. Kahneman D. Thinking, Fast and Slow. 1st Pbk. ed. New York: Farrar, Straus and Giroux; 2013 